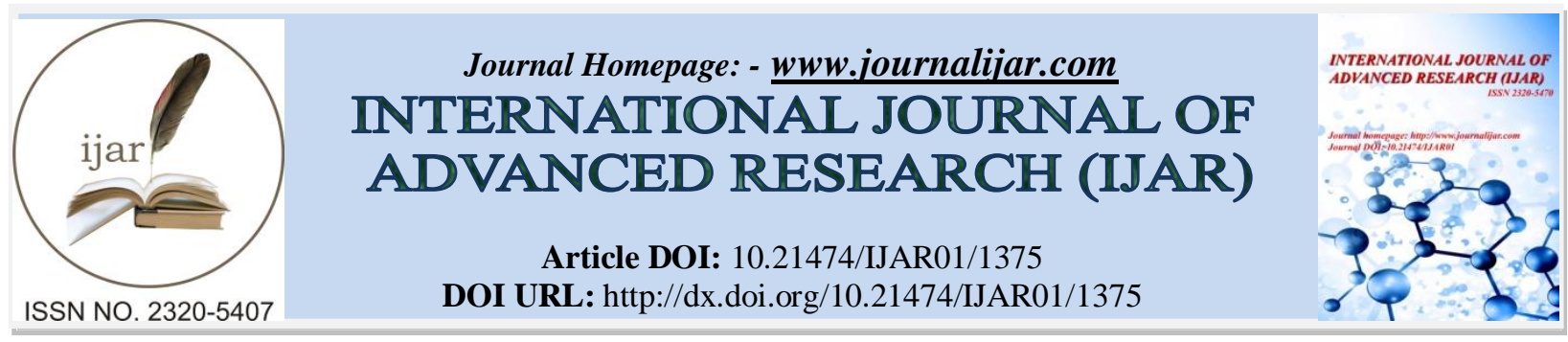

RESEARCH ARTICLE

\title{
DIFFERENTIAL CHANGES IN ISOZYME PROFILES OF ANTIOXIDANT ENZYMES INDUCED BY PESTICIDES AND NEEM ALONE AND IN COMBINATION IN TOMATO PLANTS (LYCOPERSICON ESCULENTUM).
}

*Vandita Sinha and P.K.Tandon.

Department of Botany, University of Lucknow, Lucknow-7, UP, India.

\section{Manuscript Info}

Manuscript History

Received: 12 June 2016

Final Accepted: 19 July 2016

Published: August 2016

Key words:-

Isozymes, Antioxidants, Neem,

Dimethoate, Cypermethrin, Tomato

\begin{abstract}
Changes in isozyme profiles of antioxidants SOD, CAT, APX, GPOX and GR in tomato plants under the effect of individual doses of two pesticides dimethoate $(\mathrm{D} 1=0.1 \%$ and $\mathrm{D} 2=0.2 \%)$ and cypermethrin $(\mathrm{C} 1=0.03 \%$ and $\mathrm{C} 2=0.06 \%)$ and biopesticide neem $(\mathrm{N} 1=0.4 \%)$ as well as combined doses of dimethoate/cypermethrin and neem $(\mathrm{D} 1+\mathrm{N} 1, \mathrm{D} 2+\mathrm{N} 1, \mathrm{C} 1+\mathrm{N} 1, \mathrm{C} 2+\mathrm{N} 1)$ were analysed through Native PAGE. Application of neem resulted in lower SOD and better CAT activity besides coordination among the activities of other antioxidants. Intensity of SOD bands increased whereas inhibition in CAT expression was observed with increasing pesticide doses suggesting higher production of $\mathrm{O}_{2}^{-}$. SOD1 and SOD3 were found to be associated with dimethoate toxicity whereas SOD2 was linked with cypermethrin toxicity. Dimethoate stress inhibited APX enzyme expression whereas APX was sensitive to higher dose of Cypermethrin. An exclusive role of GPOX2 isozyme was found in total peroxidase activity in tomato plants. GPOX was found to be more sensitive to dimethoate whereas GR was found to be more sensitive to Cypermethrin. At combined doses, low intensity SOD bands and high CAT activity band was observed which might be associated with lower generation and higher detoxification of $\mathrm{O}_{2}^{-}$. Further, activities of APX, GPOX and GR were also found to be coordinated and stable at combined doses exhibiting enhancement in antioxidant capacity in tomato plants due to supplementation of neem.
\end{abstract}

\section{Introduction:-}

Plants are exposed to various natural and environmental stresses, which affect their physiology, morphology, and development and cause considerable losses in the productivity of many crops (Hung et al., 2005). When plants are subject to stress, whether biotic or abiotic, increased production of potentially dangerous active Oxygen species is favoured (Asada and Takahashi, 1987; Wise and Naylor, 1987). Plants are able to adapt to adverse conditions, to some extent, through altered rates of protein synthesis or repair and through amelioration of their defensive capacity owing to increases in endogenous levels of antioxidative enzymes (Edwards et al., 1994; Mittler and Zilinskas, 1994). 
In recent years efforts have been initiated to enhance plant tolerance to oxidative stress by modifying the plant antioxidant defense system through molecular biological techniques (Foyer et al., 1994; Allen, 1995).

Isozyme, as the product of the gene expression, could be a biochemical criterion to know the tolerance of plant to stress besides detecting the inheritance and variance at the molecular level. Recently, many studies had shown close correlation between isozymes of plant and their tolerance to disease and stress (Ying et al., 2006).

It is strongly believed that changes induced by oxidative stress in the activities of antioxidant enzymes could be due to the synthesis of new isoforms (Edwards et al., 1994). To investigate changes in the isoforms of SOD, POD, GR, and AP in plants, proteins are subjected to Native Polyacrylamide Gel Electrophoresis (PAGE) and stained for their activities. Different patterns of electrophoretic isoenzyme are always correlated with the difference of relative gene expression. The different enzyme bands in cell are produced to adapt to different cellular circumstance. The variability of one enzyme band, at the same adversity, may be isochronous with another, even reverse (Ying et al., 2006). Since equal amounts of protein extracts are electrophoresed in a nondenaturing gel, the band intensity equates to enzyme activity (Kuk et al., 2003).

Pesticides that are being actively used to boost agriculture to meet the demand of the growing population have both positive and negative effects (Sinha et al., 2009). Pesticides generally induce changes in the physiological and biochemical processes of the plants which could further result into growth retardation, change in nutritional status as well as antioxidant potential of plant (Van Eerd et al., 2003). Neem based pesticides are safe, non-toxic \& do not keep any residual effects on agricultural produce. Neem, having plant growth promoting and pesticidal effect, is being used in combination with other pesticides. Neem has proved to be beneficial on plants with almost negligible phytotoxicity; hence neem may play a protective role against the damages caused by the pesticides and other stresses on plants (Gandhi et al., 2006).

Several studies have been done to study the effect of different stresses on expression of isozymes (El-baky et al., 2003; Anderson et al., 1995; Rao et al., 1995). Research, which focuses on isozymes expression in plants due to pesticide stress is comparatively rare and the interactive effects of pesticide stress and neem on isozymes expression has not been done so far.

In this context, the present study was aimed to assess comparative and combined effects of neem and pesticide on isozymes of tomato plants (Lycopersicon esculentum) through Native Polyacrylamide Gel Electrophoresis (PAGE). The correlation between different treatments and the five isozymes, viz. SOD, CAT, APX, GPOX and GR, of the antioxidant system in the plants was studied by analyzing the difference between intensities in electrophoretograms through Native Polyacrylamide Gel Electrophoresis (PAGE).

Tomato plants were subjected to two sets of treatments under standard glasshouse conditions, first with pesticide dimethoate and second with pesticide cypermethrin. The doses of neem, dimethoate and cypermethrin were selected as per recommended field practices (RFP) and after further screening. Seed germination/root elongation test was performed to screen out the dose of neem to be supplemented with dimethoate and cypermethrin. Treatments given in the first set were control $(\mathrm{C})$, neem $(\mathrm{N} 1=0.4 \%)$, dimethoate $(\mathrm{D} 1=0.1 \%$ and $\mathrm{D} 2=0.2 \%)$ and combined doses of dimethoate and neem $(\mathrm{D} 1+\mathrm{N} 1$ and $\mathrm{D} 2+\mathrm{N} 1)$. Similarly treatments given under second set with cypermethrin were C, $\mathrm{N} 1, \mathrm{C} 1(0.03 \%), \mathrm{C} 2(0.06 \%), \mathrm{C} 1+\mathrm{N} 1$ and $\mathrm{C} 2+\mathrm{N} 1$.

\section{Materials and methods:-}

The effects of pesticide stress alone as well as in combination with neem on the electrophoretic pattern of various enzymes viz. SOD, CAT, APX, GPOX, GR were studied. Enzyme extracts were prepared as required for the assay of respective enzyme. Enzyme samples were subjected to Native Polyacrylamide Gel Electrophoresis (PAGE) following the method of Laemmli, (1970) with some modifications using $0.01 \mathrm{M}$ Tris-glycine buffer ( $\mathrm{pH} \mathrm{8.3)} \mathrm{as}$ running buffer (with no SDS), $10 \%$ resolving \& 3.5\% stacking gel for SOD, APX, GPOX, GR and 7\% resolving \& $3 \%$ stacking gel for CAT. Isozymes were separated on nondenaturing polyacrylamide gels. In gel activity staining for different enzymes was done. Enzyme samples with equal quantity of protein along with loading buffer (with no $\beta$-mercaptoethanol) were layered on the top of the stacking gel and electrophoretic run was completed using a current of $25 \mathrm{~mA}$ per slab at $80 \mathrm{~V}$ and $4^{\circ} \mathrm{C}$ using SDS PAGE Assembly, Gehoeffer, GE Healthcare. Densitometry was done using Quantity One Software with Geldoc System (Biorad). 


\section{Isozymes of Superoxide Dismutase (SOD):-}

After electrophoresis, gels were stained with a method of Beauchamp and Fridovich, (1971) with some modifications. The gels were incubated in $50 \mathrm{mM}$ potassium phosphate buffer (pH 7.8) containing $2.5 \mathrm{mM}$ NBT in dark for $25 \mathrm{~min}$. The gels were then soaked in $50 \mathrm{mM}$ potassium phosphate buffer $(\mathrm{pH} 7.8)$ containing $30 \mu \mathrm{M}$ riboflavin and $0.4 \% \mathrm{~N}, \mathrm{~N}, \mathrm{~N} 9, \mathrm{~N} 9$-tetramethylethylenediamine (TEMED) in the dark for $40 \mathrm{~min}$. The gels were then illuminated for 10 to 15 min until the appearance of enzyme bands and were then transferred to $1 \%$ (v/v) acetic acid to stop the reaction. The SOD staining method involves formation of the blue coloured complex due to reduced NBT formed by oxygen radicals from exposure of riboflavin to white light, the SOD activity is detected by the colourless bands.

\section{Isozymes of Catalase (CAT):-}

For detection of catalase isozymes bands, the gels were soaked in $3.27 \mathrm{mM} \mathrm{H}_{2} \mathrm{O}_{2}$ for $25 \mathrm{~min}$, rinsed in water and stained in a solution of $1 \%(\mathrm{w} / \mathrm{v})$ potassium ferricyanide, $1 \%(\mathrm{w} / \mathrm{v})$ ferric chloride [equal volumes of $2 \%(\mathrm{w} / \mathrm{v})$ solutions of each component, added sequentially] in a method similar to that of Woodbury et al., (1971). The catalase staining method is a negative staining method where catalase is detected as colourless bands on a blue coloured background due to potassium ferricyanide ferrichloride complex.

\section{Isozymes of Ascorbate Peroxidase (APX):-}

For detection of ascorbate peroxidase isoforms in-gel activity staining for ascorbate peroxidase was done as described by Nakano and Asada, (1987). After electrophoresis, gels were rinsed in distilled water and incubated for $15 \mathrm{~min}$ in $0.1 \mathrm{M}$ potassium phosphate buffer ( $\mathrm{pH}$ 6.4) containing $4 \mathrm{mM}$ ascorbate and $4 \mathrm{mM} \mathrm{H}_{2} \mathrm{O}_{2}$. Gels were then rinsed with water and stained in a solution of $0.125 \mathrm{~N} \mathrm{HCl}$ containing $0.1 \%$ potassium ferricyanide and $0.1 \%$ ferrichloride $(\mathrm{w} / \mathrm{v})$. Isozymes of APX were visualised as colourless bands on a greenish-blue background (negative staining).

\section{Isozymes of Guaiacol Peroxidase (GPOX):-}

In-gel activity staining for guaiacol peroxidase was performed as described by McDougall, (1991). After electrophoresis, gels were rinsed in distilled (chilled) water and were incubated in sodium phosphate buffer (100 $\mathrm{mM}, \mathrm{pH}$ 7.0). The peroxidase bands were visualised by incubating the gel in a solution containing guaiacol (10 $\mathrm{mM})$ and $\mathrm{H}_{2} \mathrm{O}_{2}(0.2 \%)$. Isozymes were visualized as brick-red bands.

\section{Isozymes of Glutathione Reductase (GR):-}

After electrophoresis, gels were stained with a method of Anderson et al., (1990) with some modifications. Glutathione reductase activity was detected on native-PAGE gels by incubating them in $100 \mathrm{~mL}$ solution containing $10 \mathrm{mg}$ MTT, $10 \mathrm{mg}$ 2,6-dichlorophenolindophenol, $3.4 \mathrm{mM} \mathrm{GSSG}, 0.4 \mathrm{mM} \mathrm{NADPH}$, and $50 \mathrm{mM}$ Tris (pH 7.5). Duplicate gels were also stained in the absence of GSSG to distinguish GR from other sources of MTT reduction.

\section{Results and Discussion:-}

Superoxide Dismutase (SOD):-

Superoxide dismutase (SOD) is an important enzyme of the antioxidant system and generally exists in all kinds of plants. Native PAGE revealed three isoforms exhibiting SOD activity under all the treatments (Fig 1a and 2a). Expression of these proteins bands were genetically regulated, depending on the pesticide concentration. Three distinct SOD isozyme bands were observed in sugarcane leaves exposed to paraquat stress (Chagas et al., 2008).

SOD activity was low in control and neem plants as reflected by low band intensity of all the three isozymes which might be due to less production of toxic $\mathrm{O}_{2}{ }^{-}$in control and neem exposed plants (Fig 1a and 2a).

The activities of all the isozymes increased with the increase in concentration of pesticides. Under the set of plants exposed to dimethoate treatments (Fig 1a) bands of SOD2 were slightly faded as compared to bands of SOD1 and SOD3. It appears that contribution of SOD2 was less in comparison to other SOD isozymes in total activity of SOD. Intensity of SOD3 isozyme was higher in case of dimethoate doses D1 and D2 (Lane 3 and 4) whereas in case of combined doses D1+N1 and D2+N1 (Lane 5 and 6) intensity of SOD1 isozyme was higher (Fig 1a). At D2 dose (Lane 4) intensity of SOD bands was highest at all the isoforms; hence total band intensity in Lane 4 (D2 treated plants) was highest. Under the set of plants exposed to cypermethrin treatments (Fig 2a) SOD1 contributed more in total activity at C1 (Lane 3) whereas SOD2 was more prominent at C2 dose (Lane 4). It could be inferred that the SOD2 might be correlated with cypermethrin toxicity and was induced by high cypermethrin concentration. It 
appears that there is preferential expression and role of these isozymes in detoxification of $\mathrm{O}_{2}{ }^{-}$. Consequently these high intensity bands indicated that pesticide stress induced related gene(s) to produce these inducible proteins. Enhance $\mathrm{O}_{2}^{-}$production has been attributed as the cause of increase in the total SOD activity in the stressed genotypes (Asada and Takahashi, 1987). It was found that oxidative stress caused by acetamiprid in bacteria may not only elevated SOD activity but also generated new SOD isozymes (Yao et al., 2006).

Total band intensity of all three isozymes were found to be decreased in plant exposed to combined treatment of pesticide and neem (Lane 5 and 6) in comparison to the individual pesticide doses (Lane 3 and 4). Increased expression of SOD1 was found at these doses as compared to SOD2 and SOD3 (Fig 1a and 2a). Descending SOD activity under neem and combined treatments were found in enzyme activity analysis also. Increase in SOD activity in pesticide alone dose might be due to the stress imposed by pesticide on plants which might be due to an increase in superoxide anions.

At least five or six isoforms were found in the shoot and root of barley due to saline stress with SOD1 and SOD5 being the major ones and examined the increase in enzyme activity coincided with a variable increase in the individual isoform expression (Kim et al., 2005).

\section{Catalase (CAT):-}

The electrophoretic patterns of catalase isozymes showed one high intensity isozyme CAT1 with different intensities and expressions under any of the pesticide treatments (Fig $1 \mathrm{~b}$ and $2 \mathrm{~b}$ ). It was found that onion cultivars when grown under control and salt stress treatments, each cultivar exhibited one band of CAT as a total number with different intensity (El-baky et al., 2003). High intensity CAT activity bands were found in control and neem treatments (Lane 1 and 2 respectively). However catalase activity was found to be better in neem treated plants in relation to control.

The changes in CAT isozymes under different treatments were quite different to that of SOD isozymes. CAT activity was significantly descended in tomato under dimethoate stress in comparison to control as evident by lower intensity bands at Lane 3 and 4 (Fig 1b). The activity of CAT decreased along with the increasing concentration of pesticide and band intensity in D2 treated plants (Lane 4) was drastically reduced. Decline in CAT was also observed in plants treated with Cypermethrin dose $\mathrm{C} 1$ and $\mathrm{C} 2$ (Lane 3 and 4) with concomitant increase in the dose (Fig 2b). It indicated that the CAT isozyme of tomato plants was relatively sensitive to both dimethoate and cypermethrin treatments. Here role of other enzymes for $\mathrm{H}_{2} \mathrm{O}_{2}$ detoxification become important due to decreased expression of CAT isozymes or CAT activity. The intensity of CAT isozyme bands in Onion cultivars when grown under salt stress treatments was decreased with increasing salt treatment (El-baky et al., 2003). Studies have shown that CAT activity rapidly declines in response to chilling in combination with high light (Feierabend et al., 1992; Mishra et al., 1993), likely a result of inactivation of CAT by active oxygen (Feierabend and Engel, 1986).

A higher induction of CAT was found when plants were exposed to combined doses of pesticide and neem (Lane 5 and 6) in comparison to pesticide alone doses (Lane 3 and 4) (Fig 1b and 2b). It is noteworthy that CAT activity was maintained in the combined treatment, since prevention of loss of activity may be as important for plant against oxidative stress as induction Higher CAT activity might have helped in reducing toxic $\mathrm{H}_{2} \mathrm{O}_{2}$ species exhibiting the ameliorative effect of neem on stress induced by dimethoate and cypermethrin doses in tomato and thus would have resulted in better protection. Higher CAT activity in both leaves and roots and high activity of CAT isozymes in cold-acclimated leaves of Rice suggests a more efficient scavenging of $\mathrm{H}_{2} \mathrm{O}_{2}$, for better protection against this toxic molecule during chilling and recovery (Kuk et al., 2003).

\section{Ascorbate Peroxidase (APX):-}

The electrophoretogram of tomato showed three isozyme bands APX1, APX2, and APX3 under all the treatments, however these bands were found to be weak and sensitive to dimethoate doses D1 and D2 (Fig 1c). It appears that dimethoate stress inhibited APX enzyme activity. Three isoforms were observed in wheat plants grown under ambient or high $\mathrm{CO}_{2}$. AP-1 disappeared in plants grown under $\mathrm{O}_{3}$, and the staining intensities of AP-2 and AP-3 decreased significantly (Rao et al., 1995). Under different treatments, both the number and activity of APX isozyme bands were found to be different in expression. Bands of APX2 were relatively weaker than APX1 and APX3 at all the treatments other than combined doses (Lane 5 and 6) at which APX3 was of lowest intensity.

Under dimethoate treatments intensity of APX was higher at D2 and D2+N1 dose than that at D1 and D1+N1 dose respectively (Fig 1c). Also APX isozymes showed enhanced intensities under combined treatments of pesticide and neem $(\mathrm{D} 1+\mathrm{N} 1$ and $\mathrm{D} 2+\mathrm{N} 1)$ in comparison to pesticide alone doses (D1 and D2). Here APX1 was actively strong 
and contributed maximum in the total APX activity in combined treatments. It seems that neem has altered APX activity when combined with dimethoate treatments and protected the plants against dimethoate induced damage by enhancing APX activity. Similar results were reported in wheat plants that combination of high $\mathrm{CO}_{2}$ and $\mathrm{O}_{3}$ appeared to have preferentially enhanced the staining intensities of AP-2, and AP-3 and were capable of resisting $\mathrm{O}_{3}$ induced damage to growth and proteins compared to plants exposed to $\mathrm{O}_{3}$ under ambient $\mathrm{CO}_{2}$ (Rao et al., 1995).

Under Cypermethrin treatments APX3 was of high intensity in Control, N1 and C1 treatments. At C2 dose, APX3 though appeared but was of reduced activity (Lane 4, Fig 2c). However APX3 activity was negligible at C1+N1 and $\mathrm{C} 2+\mathrm{N} 1$ dose as evident by fainted bands at these treatments (Lane 5 and 6) (Fig 2c). Induction of only cytosolic isoforms was found under salt stress in leaves of rice plants (Lee et al., 2001). APX isozymes were found to be exceptionally induced in plants exposed to lower dose of cypermethrin i.e. C1 (Lane 3, Fig 2c). Total intensity of APX bands was greater at C1 dose (Lane 3) due to concomitant increase in APX1 and APX3 expressions. However at C2 dose (Lane 4) this activity was lower than that in control (Lane 1). APX activity was found to be decreased at combined treatments. APX1 and APX2 isozyme were active in $\mathrm{C} 1+\mathrm{N} 1$ and $\mathrm{C} 2+\mathrm{N} 1$ treated plants but due to negligible APX3 activity in these treatments total activity was reduced (Lane 5 and 6). APX1 was highly reduced at $\mathrm{C} 1+\mathrm{N} 1$ dose as compared to that at its individual dose $\mathrm{C} 1$ and also at control. APX2 activity was found to be increased at $\mathrm{C} 2+\mathrm{N} 1$ dose in comparison to that at $\mathrm{C} 2$ and control (Fig 2c). It appears that all the three APX isozyme activities were selectively active to different treatments. Similarly, it was reported that despite the fact that total APX activity had no modification, their isozymes were clearly affected demonstrating a selective response of each isoform (Yannarelli et al., 2006).

\section{Guaiacol Peroxidase (GPOX):-}

Zymograms of GPOX showed two isozymes GPOX1 and GPOX2 where GPOX2 was more prominent (Fig 1d and $2 \mathrm{~d})$. This suggested an exclusive role of GPOX2 isozyme in total peroxidase activity in tomato plants. Control showed lowest peroxidase activity as revealed by band densities. Expressions of isozymes were increased in plants exposed to both dimethoate and cypermethrin when compared to that in control and neem treated plants (Fig 1d and 2d). This revealed that pesticide treatments have increased the peroxidase activity and accumulation of these isozymes encoding gene(s) which might have accelerated in response to pesticide stress. The density and intensity of POD band was higher compared to the control in the salt treated Onion Giza 20 cultivars (El-baky et al., 2003). A higher level of GPOX was correlated with lower level of APX activity in D1 treated plants in comparison to that in D2 treated plants (Fig 1d). Under cypermethrin treatments, at C2 dose both isozymes GPOX1 and GPOX2 were very prominent (Lane 4) however GPOX2 was over expressed in C2 treated plants and contributed more in total isozyme activity (Fig 2d). These results indicated that cypermethrin stress increased the accumulation of the GPOX enzyme. Here again A higher level of GPOX might be correlated with lower level of APX activity in C2 treated plants in comparison to $\mathrm{C} 1$ treated plants (Fig 2d).

Under dimethoate treatments intensities of both the isozymes at combined doses (Lane 5 and 6) were though lower than the intensities at the respective individual doses (Lane 3 and 4 respectively) yet found to be relatively higher than that in control and neem treated plants (Lane 1 and 2). In combined treatments of cypermethrin and neem GPOX activity was increased at $\mathrm{C} 1+\mathrm{N} 1$ as shown in Lane 5, Fig 2d. These results were in harmony with the findings that the growth of wheat plants under a combination of high $\mathrm{CO}_{2}$ and $\mathrm{O}_{3}$ preferentially enhanced the intensity of POD-1 (Rao et al., 1995). However C2+N1 treated plants showed a decrease in the enzyme expression in both the isoforms with respect to $\mathrm{C} 2$ treated plants (Fig 2d). It seems there is differential expression of GPOX in accordance with other isozymes. Under water stress, the activities and isozyme bands of SOD, POD and CAT all changed, out of which the changes of bands and activities of peroxidase isozymes were more significant (Sun et al., 2003).

\section{Glutathione Reductase (GR):-}

There appeared two GR isozyme bands, namely GR1 and GR2 under all treatments where intensity and expression of GR2 was found to be higher. Band intensity was highest in control and neem treated plants comparison that in plants treated with any other treatments (Fig 1e and 2e). Dimethoate doses showed decrease in total GR activity in comparison to control however total GR activity was higher at D2 (Lane 4) than that at D1(Lane 3) which may be attributed to higher GR1 expression at D2 dose (Fig 1e). Stress conditions are frequently found to induce increase in the extractable activities of free radical-metabolizing enzymes such as GR and other protective enzymes (Foyer et al., 1991). Intensity of GR1 bands at control, neem and C1 dose (Fig 2e, Lane 1, 2 and 3 respectively) was exceptionally higher than that at other three treatments viz. $\mathrm{C} 2, \mathrm{C} 1+\mathrm{N} 1$ and $\mathrm{C} 2+\mathrm{N} 1$ (Lane 4, 5 and 6 respectively). 
GR1 and GR2 isozyme activities were found to be decreased with increasing cypermethrin concentration and this decrease was more prominent in GR1 isozyme. It appears that GR1 was more sensitive to higher dose of pesticide i.e. $\mathrm{C} 2$ at which the activity of GR1 was lowest among the test doses (Fig 2e).

A decrease in intensities of both the GR isozyme bands was observed at D2+N1 dose (Fig 1e, Lane 6) in respect to that at the individual D2 dose (Lane 4). However an increase in total GR bands was observed at D1+N1 dose (Lane 5) in respect to that at individual D1 dose (Lane 3) that too mainly because of GR1 bands. This change in expression of the enzyme would probably be due to differential and coordinated expression of GR isozyme with other enzymes. It was suggested that plants exposed to $\mathrm{O}_{3}$ under high $\mathrm{CO}_{2}$, are capable of differentially altering the isoform composition of GR and capable of protecting plant growth from $\mathrm{O}_{3}$ induced oxidative damage in comparison to $\mathrm{O}_{3}$ alone (Rao et al., 1995). Intensity of GR bands was found to be lower at combined doses of cypermethrin and neem as compared to that at individual pesticide doses (Fig 2e). Intensity of GR1 band was higher at C2+N1 dose (Fig 2e, Lane 6) in comparison to that at $\mathrm{C} 1+\mathrm{N} 1$ dose (Lane 5) whereas intensity of GR2 band was slightly higher at $\mathrm{C} 1+\mathrm{N} 1$ dose than that at $\mathrm{C} 2+\mathrm{N} 1$ dose. The net effect of this was seen as higher GR activity at $\mathrm{C} 2+\mathrm{N} 1$ dose than that at $\mathrm{C} 1+\mathrm{N} 1$ dose though non significantly. Similarly GR profile was observed in the maize mesocotyl in response to acclimation and chilling (Anderson et al., 1995). The upper band was greatly diminished in the acclimation and acclimation chilling treatments, whereas the lower two bands were greatly increased. The net effect was the absence of a significant change in total GR activity (Anderson et al., 1995).

\section{Conclusion:-}

- Different isozymes/bands of an enzyme showed differential change in intensities under various stresses.

* Low intensity SOD bands and high intensity bands of other antioxidants mainly CAT, APX and GR were found in control and neem treated plants.

* Intensity of SOD isozymes increased under pesticide stress showing enhanced $\mathrm{O}_{2}{ }^{-}$production whereas CAT activity decreased under increasing pesticide concentrations.

* Isozymes of APX, GPOX and GR showed differential response to different treatments.

- Bands of APX were found to be weak at both the dimethoate doses D1 and D2. APX activity was exceptionally induced at lower dose of cypermethrin $\mathrm{C} 1$ but reduced drastically at its higher dose $\mathrm{C} 2$. It could be inferred that both the doses of dimethoate inhibited APX activity whereas APX was sensitive to only higher dose of Cypermethrin.

- Exclusive role of GPOX2 isozyme was found in total peroxidase activity in tomato plants at all the doses. Reduced GPOX activity and higher GR activity was observed at higher dose of dimethoate as compared to its lower dose whereas higher GPOX activity and lower GR activity was observed at higher dose of cypermethrin as compared to its lower dose. Thus GPOX was found to be more sensitive to dimethoate whereas GR was found to be more sensitive to Cypermethrin.

* Intensity of SOD bands was found to be lower at combined doses of pesticide and neem as compared to that at individual pesticide doses whereas induction of expression and activity was observed in CAT bands at combined doses of pesticide and neem. This indicated that on the one hand production of $\mathrm{O}_{2}{ }^{-}$was lower at combined doses and on the other hand whatever $\mathrm{O}_{2}{ }^{-}$was produced was detoxified at a higher rate. Activities of other antioxidants were also found to be more coordinated and stable at combined doses.

* We deduce that SOD and CAT are the most important and primary enzymes for the signaling and control of pesticide stress in tomato plants and neem when applied concomitantly with pesticide was not only able to reduce the toxic effects of pesticides but these combinations were also found to be effective in increasing the antioxidant potential in tomato plants.

Above results suggested that pesticide toxicity might be mediated by oxidative stress and better protection of the tomato plants from this oxidative damage was achieved due to supplementation of neem which resulted in coordinated activity of the antioxidant system by inducing selective adaptive responses in affected Plants. Thus the ability to increase the preventive and coordinated antioxidant system in order to limit cellular damages and improve plant tolerance against pesticide might be an important role of neem. 


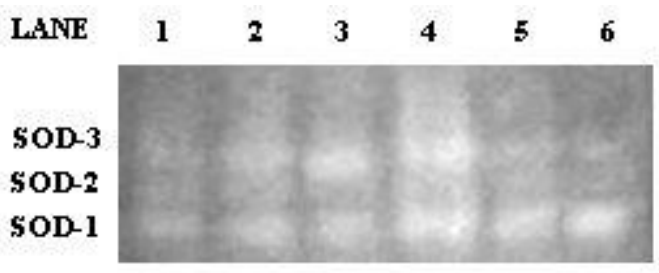

la) SOD-Dimethoate

CAT-1

lb) CAT-Dimethoate

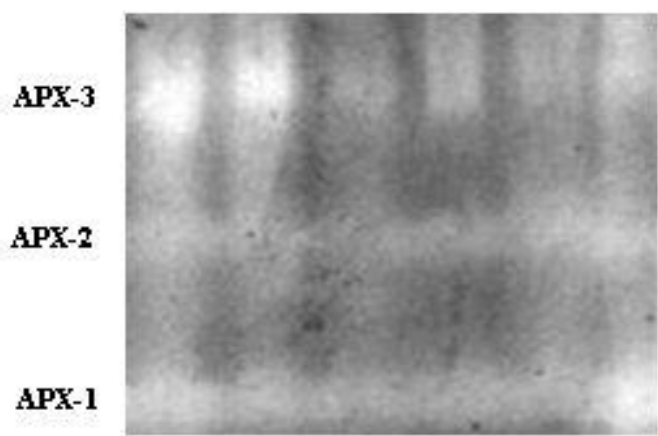

1c) APX-Dimethoate

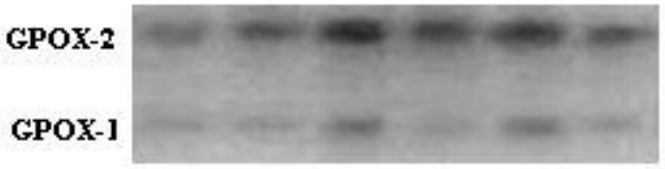

1d) GPOX-Dimethoate

GR-2

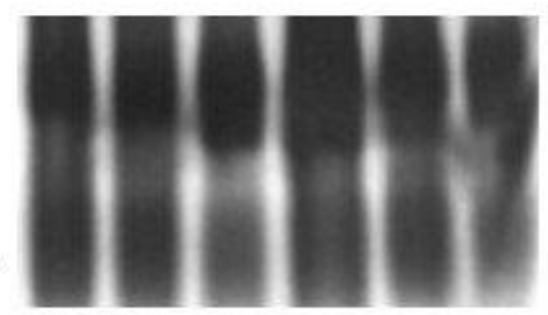

le) GR-Dimethoate

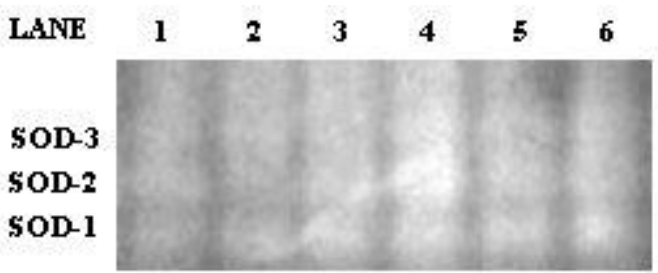

2a) SOD-Cypermethrin

CAT-1

2b) CAT-Cypermethrin

APX-3

APX-2

APX-1

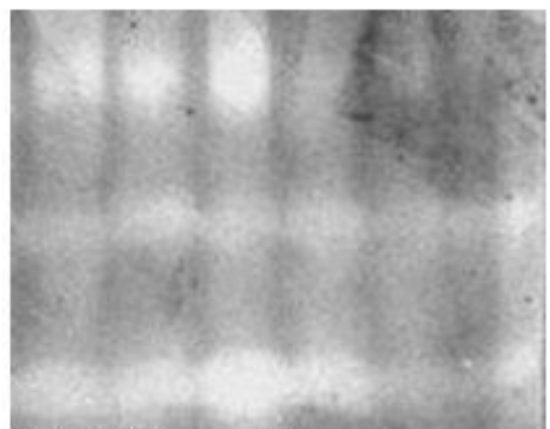

2c) APX-Cypermethrin

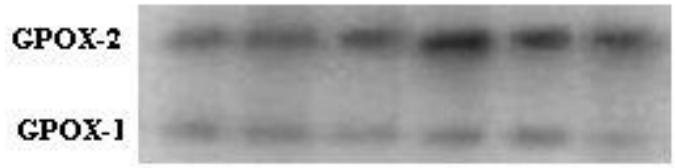

2d) GPOX-Cypermethrin

GR-2

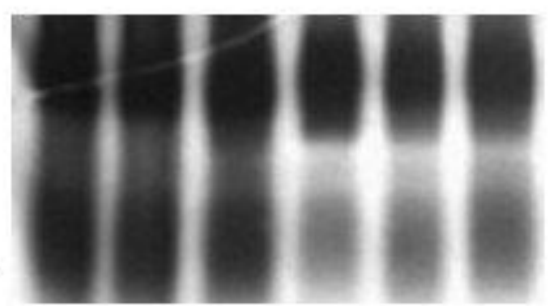

2e) GR-Cypermethrin

Figure 1:- Effect of neem, dimethoate and their combined doses on isozymes of various antioxidant enzymes in tomato plants. Lane 1 = Control, Lane $2=\mathrm{N} 1$, Lane 3 $=\mathrm{D} 1$, Lane $4=\mathrm{D} 2$, Lane $5=\mathrm{D} 1+\mathrm{N} 1$ and Lane $6=\mathrm{D} 2+\mathrm{N} 1$ where $\mathrm{N} 1=0.4 \%$ Neem, D1 \& D2 $=0.1 \%$ \& $0.2 \%$ Dimethoate respectively. 


\section{References:-}

1. Allen, R.D. (1995): Dissection of oxidative stress tolerance using transgenic plants. Plant Physiol., 107: 10491054.

2. Anderson, J.V., Hess, J.L. and Chevone, B.I. (1990): Purification, characterization, and immunological properties for two isoforms of glutathione reductase from eastern white pine needles. Plant Physiol., 94: 14021409.

3. Anderson, M. D., Prasad, T. K., and Stewart, C. R. (1995): Changes in isozyme Profiles of Catalase, Peroxidase, and Glutathione Reductase during Acclimation to Chilling in Mesocotyls of Maize Seedlings. Plant Physiol., 109: 1247-1257.

4. Asada, K., and Takahashi, M. (1987): Production and scavenging of active Oxygen in Photosynthesis. In: Kyle Z.D.J., Osmond C.B., and Arntzen C.J. (eds) Photoinhibition: Elsevier Science, Amsterdam, The Netherlands. pp. 227-287.

5. Beauchamp, C. and Fridovich, I. (1971): Superoxide dismutase: improved assays and an assay applicable to acrylamide gels. Anal. Biochem., 44: 276-287.

6. Chagas, R.M., Silveirab, J.A.G., Ribeiroc, R.V., Vitorellod, V.A., and Carrera, H. (2008): Photochemical damage and comparative performance of superoxide dismutase and ascorbate peroxidase in sugarcane leaves exposed to paraquat-induced oxidative stress. Pest. Biochem. Physiol., 90(3): 181-188.

7. Edwards, E.A., Enard, C., Creissen, G.P., and Mullineaux, P.M. (1994): Synthesis and properties of glutathione reductase in stressed peas. Planta, 192: 137-143.

8. El-baky, A., Hanaa, H., Amal Mohamed, A., and Hussein, M.M. (2003): Influence of Salinity on Lipid Peroxidation, Antioxidant Enzymes and Electrophoretic Patterns of Protein and Isoenzymes in Leaves of Some Onion Cultivars. Asian J. Plant Sci., 2(17-24): 1220-1227.

9. Feierabend, J., and Engel, S. (1986): Photoinactivation of catalase in vitro and in leaves. Arch Biochem Biophys., 251: 567-576.

10. Feierabend, J. Schaan, C., and Hertwig, B. (1992): Photoinactivation of catalase occurs under both high and low-temperature stress conditions and accompanies photoinhibition of photosystem II. Plant Physiol., 100: 1554-1561.

11. Foyer, C.H., Descourvieres, P., and Kunert, K. J. (1994): Protection against oxygen radicals: an important defense mechanism studied in transgenic plants. Plant Cell Environ., 17: 507-523.

12. Foyer, C., Lelandais, M., Galap, C., and Kunert, K.J. (1991): Effects of Elevated Cytosolic Glutathione Reductase Activity on the Cellular Glutathione Pool and Photosynthesis in Leaves under Normal and Stress Conditions. Plant Physiol., 97: 863-872.

13. Gandhi, Indira, P., Gunasekaran, K., and Sa, Tongmin. (2006): Neem oil as a potential seed dresser for managing Homopterous sucking pests of Okra Abelmoschus esculentus (L.) Moench. J. Pest. Sci., 79: 103-111.

14. Hung, S.H., Yu, C.W., and Lin, C.H. (2005): Hydrogen peroxide functions as a stress signal in plants. Bot. Bull. Acad. Sin., 46: 1-10.

15. Kim, S. Y., Lim, J-H., Park, M. R., Kim, Y. J., Park, T.I., Seo, Y. W., Choi , K. G., and Yun, S. J. (2005). Enhanced Antioxidant Enzymes are Associated with Reduced Hydrogen Peroxide in Barley Roots under Saline Stress. J. Biochem. Mol. Biol., 38(2): 218-224.

16. Kuk, Y. I. S., Burgos, N. R., Hwang, T.E., Han, O., Cho, B. H., Jung, S., and Guh, J.O. (2003): Antioxidative Enzymes Offer Protection from Chilling Damage in Rice Plants. Crop Sci., 43: 2109-2117.

17. Laemmli, U.K. (1970): Cleavage of structural proteins during the assembly of the head of bacteriophage T4. Nature, 227: 680-685.

18. Lee, D.H., Kim, Y.S., and Lee, C.B. (2001): The individual response of the antioxidant enzymes by salt stress in rice (Oryza sativa L.). J. Plant Physiol., 158: 737-745.

19. McDougall, G. J. (1991): Cell wall associated peroxidases and lignification during growth of flax fibres. J. Plant Physiol., 139: 182-186.

20. Mishra, N.P., Mishra, R.K., and Singhal, G.S. (1993): Changes in the activities of anti- oxidant enzymes during exposure of intact wheat leaves to strong visible light at different temperatures in the presence of protein synthesis inhibitors. Plant Physiol., 102: 903-910.

21. Mittler, R. and Zilinskas, B.A. (1994): Regulation of pea cytosolic ascorbate peroxidase and other antioxidant enzymes during the progression of drought stress and following recovery from drought. Plant J., 5: 397-405.

22. Nakano, J., and Asada, K. (1987): Purification of ascorbate peroxidase in spinach chloroplasts: its inactivation in ascorbate depleted medium and reactivation by monodehydroascorbate radical. Plant Cell Physiol., 28: 131140 . 
23. Rao, M.V., Hale, B.A. and Ormrod, D.P. (1995): Amelioration of Ozone-induced Oxidative Damage in Wheat Plants Grown under High Carbon Dioxide' Role of Antioxidant Enzymes. Plant Physiol., 109: 421-432.

24. Sinha, V., Rai, V. and Tandon P.K. (2009): Pesticides: Use, Impact and Regulations for Management. In Ashworth G.S. and Azevedo P., (eds) Agricultural Wastes. Nova Science Publishers, Inc., New York, pp. 93107.

25. Sun C.X., Liu, Z.G., and Jing, Y.D. (2003): Effect of stress on activity and isozyme of the major defenseenzyme in maize leaves. J. Maize Sci., 11: 63-66.

26. Van Eerd, Laura L., Hoagland, Robert E., Zablotowicz, Robert M., and Christopher Hall, J. (2003): Pesticide metabolism in plants and microorganisms. Weed Science, 51: 472-495.

27. Wise, R.R., and Naylor, A.W. (1987): Chilling-enhanced peroxidation: the peroxidative destruction of lipids during chilling injury to photosynthesis and ultrastructure. Plant Physiol., 83: 272-277.

28. Woodbury W., Spencer A.K., and Stahmann M.A. (1971): An improved procedure using ferricyanide for detecting catalase isozymes. Anal Biochem., 44: 301-305.

29. Yannarelli, G.G., Gallego, S.M., and Tomaro, M.L. (2006): Effect of UV-B radiation on the activity and isoforms of enzymes with peroxidase activity in sunflower cotyledons. Environ. Exp. Bot., 56: 174-181.

30. Yao, X.H., Min, H. and Lv, Z.M. (2006): Response of Superoxide Dismutase, Catalase, and ATPase Activity in Bacteria Exposed to Acetamiprid. Biomed. Environ. Sci., 19: 309-314.

31. Ying, X.F., Liu, P., and Xu, G.D. (2006): Effect of aluminum on the isozymes of the seedlings of two soybeans Glycine max (L.) Merrill varieties. Plant Soil Environ., 52(6): 262-270. 